Noname manuscript No.

(will be inserted by the editor)

Ilya Mandel • M. Coleman Miller •

Bobomurat J. Ahmedov - Cosimo

Bambi · Christopher P. L. Berry .

Jeandrew Brink - Duncan Brown .

Eliana Chaverra • A. I. Chugunov •

Stephen Fairhurst - Chris Fryer •

Jonathan R. Gair · Dorota

Gondek-Rosinska - Leonardo

Gualtieri · M. E. Gusakov · Mark

Hannam - Ian Harry - E. M. Kantor .

Wlodek Kluzniak - Marcin Kucaba .

Georgios Lukes-Gerakopoulos •

H. Meheut - Andrew Melatos .

Viktoriya S. Morozova - T. Paumard .

Nikolaos Stergioulas - Anna Studzinska •

Magda Szkudlarek • Odele Straub •

G. Torok - P. Varnier - F. H. Vincent .

Mateusz Wisniewicz - M. Wildner •

Clifford Will · Kent Yagi · Olindo

Zanotti · Shuang-Yong Zhou

\title{
Relativistic Astrophysics at GR20
}

Received: date / Accepted: date

Bobomurat J. Ahmedov

Institute of Nuclear Physics, Ulughbek, Tashkent 100214, Uzbekistan

Cosimo Bambi

Department of Physics, Fudan University, Shangai, China

Christopher P. L. Berry

Institute of Astronomy, University of Cambridge, Madingley Road, Cambridge, CB3 0HA, UK; and

School of Physics and Astronomy, University of Birmingham, Edgbaston, Birmingham, B15 2TT, UK

Jeandrew Brink

National Institute for Theoretical Physics (NITheP), Bag X1 Matieland, Stellen-

bosch, 7602, South Africa; and

Physics Department, Stellenbosch University, 7602, South Africa

Duncan Brown and Ian Harry

Syracuse University, Syracuse, NY, USA 
Eliana Chaverra

Instituto de Física y Matemáticas,

Universidad Michoacana de San Nicolás de Hidalgo,

Edificio C-3, Ciudad Universitaria, 58040 Morelia, Michoacán, México.

A. I. Chugunov and M. E. Gusakov

Ioffe Physical-Technical Institute of the Russian Academy of Sciences, Polytekhnicheskaya 26, 194021 Saint-Petersburg, Russia

Stephen Fairhurst and Mark Hannam

Cardiff University, Cardiff, UK

Chris Fryer

Los Alamos National Lab, Los Alamos, NM, USA

Jonathan R. Gair

Institute of Astronomy, University of Cambridge, Madingley Road, Cambridge, CB3 0HA, UK

Dorota Gondek-Rosinska, Marcin Kucaba, Anna Studzinska, Magda Szkudlarek and Mateusz Wisniewicz

Institute of Astronomy, University of Zielona Gora, ul. Lubuska 2, PL-65-265

Zielona Gora, Poland

Leonardo Gualtieri

"Sapienza" University of Rome \& INFN, Sezione Roma 1, P.le. A. Moro 2, 00185

Roma, Italy

E. M. Kantor

Ioffe Physical-Technical Institute of the Russian Academy of Sciences, Polytekhnicheskaya 26, 194021 Saint-Petersburg, Russia; and Saint-Petersburg State Polytechnical University, Polytekhnicheskaya 29, 195251 Saint-Petersburg, Russia

Wlodek Kluzniak

Copernicus Astronomical Center, ul. Bartycka 18, PL-00-716 Warszawa, Poland

Georgios Lukes-Gerakopoulos

Theoretical Physics Institute, University of Jena, 07743 Jena, Germany

Ilya Mandel

School of Physics and Astronomy, University of Birmingham, Edgbaston, Birmingham, B15 2TT, UK

co-editor: E-mail: imandel@star.sr.bham.ac.uk

H. Meheut

CEA, Irfu, SAp, Centre de Saclay, F-91191 Gif-sur-Yvette, France

Andrew Melatos

School of Physics, University of Melbourne, Parkville VIC 3010 Australia

M. Coleman Miller 
Abstract We report the recent advances in Relativistic Astrophysics as presented at the GR20 meeting in Warsaw, Poland, in July 2013.

Department of Astronomy, University of Maryland, College Park, MD 20742 co-editor

Viktoriya S. Morozova

Max-Planck-Institut für Gravitationsphysik, Albert Einstein Institut, Am Mühlenberg 1, 14476 Potsdam, Germany

T. Paumard

LESIA, Observatoire de Paris, CNRS, Universite Pierre et Marie Curie, Universite Paris Diderot, 5 place Jules Janssen, 92190 Meudon, France

Nikolaos Stergioulas

Department of Physics, Aristotle University of Thessaloniki, Thessaloniki 54124, Greece

Odele Straub

LUTH, CNRS UMR 8102, Observatoire de Paris, Université Paris Diderot, 92190 Meudon, France

G. Torok and M. Wildner

Institute of Physics, Faculty of Philosophy and Science, Silesian University in Opava, Bezrucovo nm. 13, CZ-74601 Opava, Czech Republic

P. Varniere

AstroParticule et Cosmologie (APC), Universite Paris Diderot, 10 rue A. Domon et L. Duquet, 75205 Paris Cedex 13, France

F. H. Vincent

Copernicus Astronomical Centre, ul. Bartycka 18, 00-716, Warszawa, Poland

Clifford Will

University of Florida, Gainesville and Institut d'Astrophysique de Paris

Kent Yagi

Department of Physics, Montana State University, Bozeman, MT 59717, USA

Olindo Zanotti

Max-Planck-Institut für Gravitationsphysik, Albert Einstein Institut, Am Mühlenberg 1, 14476 Potsdam, Germany; and

Università di Trento, Laboratorio di Matematica Applicata, Via Messiano 77, I38100 Trento, Italy

Shuang-Yong Zhou

SISSA, Via Bonomea 265, 34136, Trieste, Italy and INFN, Sezione di Trieste, Italy 


\section{Introduction}

Ilya Mandel and M. Coleman Miller

The GR20 meeting which took place in July 2013 in Warsaw, Poland, included a variety of exciting presentations and posters and fruitful discussions on the topic of recent advances in Relativistic Astrophysics. The interest far outweighed the capacity of the relativistic astrophysics sessions; beyond 21 contributed talks that were given during oral sessions, 25 posters were presented.

As co-conveners and editors of this proceedings article, we opted to invite the speakers to describe their own work by contributing a brief summary to this article; we reproduce the contributions below with minimal modifications. The authors of each talk are listed after the talk title, with the presenting author italicized. Several talks are available in full at http://gr20amaldi10.edu.pl/index.php?id=18 . Because of space limitations, we could not include poster summaries here.

The summaries that follow showcase some of the continuing progress in what might be termed classical areas of relativistic astrophysics, including improving understanding of neutron-star physics and developments in modeling quasi-periodic oscillators and accretion flows.

Meanwhile, this meeting highlighted the emergence of two new roles for strong-field gravity in relativistic astrophysics. Firstly, as gravitational-wave detections are approaching, a number of talks emphasized gravity as a probe of relativistic astrophysics (see also the discussion of imminent gravitationalwave observations in the Amaldi proceedings elsewhere in this volume). Secondly, as evidenced by the talks and these proceedings, gravity, and particularly astrophysical tests of the general theory of relativity, are becoming a subject of study in their own right.

Happy reading, and we look forward to seeing you at GR-21!

\section{Nuclear Physics with Gravitational Wave Telescopes}

\section{Andrew Melatos}

Theoretical studies of bulk nuclear matter within the context of quantum chromodynamics (QCD) and effective field theories predict the existence of superfluid and superconducting phases of various types (e.g., color-flavorlocked, color-superconducting). These phases exist in the low-temperature, high-density region of the QCD phase diagram occupied by neutron stars, which is inaccessible to terrestrial experiments such as heavy-ion colliders (high temperature, low density), parity violation experiments, and giant monopole resonance experiments (low temperature, medium density). Existing astronomical data, chiefly accurate radio pulse timing and X-ray spectra of quiescent and burst emission, place some constraints on the mass, radius, and rotational evolution of neutron stars and hence the nuclear equation of state. Gravitational wave observations promise to complement the 
electromagnetic data by imaging directly the hydrodynamic motions in the superfluid, superconducting, stellar interior.

Rotational glitches in neutron stars provide a natural trigger for gravitational wave experiments. In the days to weeks following a glitch, the inviscid (neutron condensate) and viscous (proton-electron plasma) components of the superfluid in the stellar interior respond to the spin-up event and recover to a new rotational equilibrium. Theoretical analysis of radio timing data shows that the post-glitch recovery is described well as the sum of two exponentials, whose time constants reflect the mechanisms of Ekman pumping and mutual friction [4]. When the angular velocity lag between the two components is less than a critical value, an overshoot is predicted in the radio timing signature, as observed in the Crab pulsar. The same theory reproduces the results of spin-up experiments with liquid helium to $0.5 \%$ with zero free parameters. If Ekman pumping proceeds nonaxisymmetrically - a likely scenario, as the Reynolds number typically exceeds $\sim 10^{9}$, well above the threshold for nonaxisymmetric instabilities like Taylor-Gortler vortices - the post-glitch recovery is accompanied by emission of a quasimonochromatic gravitational wave signal [1], which lasts for days to weeks and can be detected in principle by pipelines developed for "long transients". A powerful multimessenger experiment is therefore possible in principle: the radio and gravitational wave data (dual polarizations) can be combined to infer the compressibility, viscosity, and stratification length-scale of bulk nuclear matter (Bennett et al. 2010), strengthening the constraints on the existence of exotic (e.g., color-flavor-locked) phases already deduced from radio data [4]. Predictions of the signal-to-noise ratio are presented in detail in Figure 3 of [1] and exceed $\sim 3$ for Advanced LIGO across a range of plausible compressibilities and viscosities.

Given the high Reynolds number expected in a neutron star, and the differential rotation which is the root driver of glitches, the superfluid interior is likely to be turbulent, even after the buoyant restoring force arising from the entropy gradient in beta equilibrium is taken into account [2]. Superfluid turbulence generates stochastic gravitational radiation. The indirect spin-down limit on individual millisecond pulsars places an upper limit of $\sim 4 \%$ on the angular velocity shear through this channel - an astrophysically significant result in its own right (see references in [2]). Moreover, the emission from every neutron star in the Universe adds together to produce a stochastic background which is detectable by Advanced LIGO if the angular velocity shear exceeds $30 \mathrm{rad} \mathrm{s}^{-1}$ averaged across the population. This is high for ordinary, isolated pulsars but plausible for millisecond pulsars, which constitute $\sim 10 \%$ of the total population [2]. If the stellar core superrotates, e.g., due to buoyancy modified Ekman pumping [3], shear-driven turbulence is even more likely to be sustained, new channels of gravitational wave emission open up (e.g., via hydrodynamic instabilities), and new multimessenger experiments involving dynamo-like magnetic activity become possible, by combining gravitational wave and radio polarization data. 


\section{Dark matter distributions around massive black holes: A general relativistic analysis}

\section{Clifford Will}

Clifford Will spoke about recent work with Laleh Sadeghian and Francesc Ferrer on how the presence of a massive black hole, such as the one at the center of our galaxy, could affect the distribution of cold dark matter [5]. The redistribution may be determined using an approach pioneered by Gondolo and Silk: [6] begin with a model distribution function for the dark matter, and "grow" the black hole adiabatically, holding the adiabatic invariants of the motion constant. Unlike the approach of Gondolo and Silk, which adopted Newtonian theory together with ad hoc correction factors to mimic general relativistic effects, Sadeghian et al. carried out the calculation fully relativistically, using the exact Schwarzschild geometry of the black hole. They found that the density of dark matter generically vanishes at $r=2 R_{\mathrm{S}}$, not $4 R_{\mathrm{S}}$ as found by Gondolo and Silk, where $R_{\mathrm{S}}$ is the Schwarzschild radius, and that the spike very close to the black hole reached significantly higher densities. They applied the relativistic adiabatic growth framework to obtain the final dark matter density for both cored and cusped initial distributions, using a Hernquist profile for the initial distribution. They showed that the gravitational effects of such a dark matter spike are significantly smaller than the relativistic effects of the black hole, including frame dragging and quadrupolar effects, for stars orbiting close to the black hole that might be candidates for testing the black hole no-hair theorems.

\section{Universal I-Love-Q Relations in Neutron Stars}

\section{Kent Yagi}

One of largest uncertainties in nuclear physics is the equation of state (EoS) in nuclear and supra-nuclear densities. Neutron-star (NS) and quarkstar (QS) observables such as the mass and radius depend strongly on the EoS $[7,8]$. We found universal relations (I-Love-Q relations) among the momentof-inertia, tidal Love number and quadrupole moment of a slowly-rotating NS and QS that are almost EoS-independent.

Such unexpected relations have several applications. On an observational astrophysical front, any one measurement of the I-Love-Q trio automatically determines the other two. On a gravitational-wave (GW) physics front, the relations help us to break degeneracy between NS spins and quadrupole moment. This allows us to determine the averaged spin of the NS binary inspiral to $\mathcal{O}(10 \%)$ with second-generation $\mathrm{GW}$ detectors such as advanced LIGO. On a fundamental physics front, any two independent measurements of the I-Love-Q trio allow for a model-independent and EoS-independent test of general relativity. For example, if one could measure the NS moment-ofinertia to $\mathcal{O}(10 \%)$ with double binary pulsar observations and the tidal Love number to $\mathcal{O}(60 \%)$ accuracy with future $\mathrm{GW}$ observations, one could place 
a constraint on a parity-violating gravitational theory that is six orders of magnitude stronger than the current bound.

\title{
5 Plasma Magnetospheres of Rotating and Oscillating Magnetized Neutron Stars in General Relativity
}

\author{
Viktoriya S. Morozova, Bobomurat J. Ahmedov, Olindo Zanotti
}

Mechanical oscillations of the neutron star crust were studied by many authors as possible explanation for a range of phenomena related to the pulsar emission, such as drifting subpulses, quasi-periodic oscillations in the spectra of soft gamma-ray repeaters, pulsar mode switching. In the recent works $[9,10]$ we attempted to investigate the mechanism by means of which the torsional oscillations of pulsar crust can influence the pulsar magnetosphere and have an imprint on the observable quantities. We have considered rotating and oscillating neutron star with zero inclination angle in frames of the space charge limited flow model and showed that the velocity of oscillations enters the Goldreich-Julian charge density, introducing additional terms to the scalar potential and accelerating electric field in the polar cap region of the pulsar magnetosphere. We found that, after decomposing the oscillation velocity in terms of spherical harmonics, the first few modes with $m=0,1$ are responsible for energy losses that are almost linearly dependent on the amplitude of the oscillation and that, for the mode $(l, m)=(2,1)$, can be a factor about 8 larger than the rotational energy losses, even for a velocity oscillation amplitude at the star surface small. We got the dependence between the product $P \dot{P}$ and the amplitude of the oscillations at the star surface, showing the influence of oscillations on the pulsar spindown.

The obtained results allowed us to propose a qualitative model for the explanation of the phenomenology of intermittent pulsars. The idea is that stellar oscillations, periodically excited by star glitches, can create relativistic winds of charged particles because of the additional electric field. When the stellar oscillations damp, the pulsar shifts below the death line in the $P$ $B$ diagram, thus entering the OFF invisible state of intermittent pulsars. Applying the obtained results to the case of generally radio-quiet magnetars we showed that the inclusion of oscillations shifts the death line downwards, allowing for the generation of radio emission from some magnetars, what is indeed supported by observations. This interpretation naturally explains the observed connection between the burst activity of magnetars and the generation of the radio emission in the magnetar magnetosphere. In addition, our results give support to the quite succesful explanation of the drifting subpulses phenomena by means of the mechanical oscillations of stellar crust, proposed in the work of Clemens and Rosen [13], as we clarified the physical mechanism for the oscillations to influence the magnetospheric processes. 


\section{Properties of differentially rotating neutron stars}

Dorota Gondek-Rosinska, Marcin Kucaba, Anna Studzinska, Magda Szkudlarek

An understanding of differentially rotating relativistic stars is key to many areas of astrophysics, in particular to the emission of gravitational waves. Newly born proto-neutron stars and compact remnants of neutron-star binary mergers are expected to rotate differentially and to be important sources of gravitational radiation. The study of differentially rotating neutron stars is a complicated task, involving the solution of Einstein's equations in a dynamical regime while, at the same time, many microphysical phenomena need to be taken into account. As a first step we have performed calculations of axisymmetric and stationary models of differentially rotating neutron stars described by a polytropic equation of state (EOS). We study rotating neutron stars for broad ranges of densities and degrees of differential rotation using a relativistic code based on multi-domain spectral methods [11]. The high level of accuracy and of stability of the code enables us for the first time to classify differentially rotating neutron stars in general relativity. We have found four distinct types of configurations. Two of these were not considered in previous works, mainly due to numerical limitations. The maximum allowed mass for the new types of configurations and moderate degree of differential rotation can be even 2-4 times higher then the maximum mass of non-rotating NSs with the same EOS [12]. Obtained results give a new view on the properties of differentially rotating neutron stars and are the starting point to studying their stability in a systematic way.

\section{Quasi-normal modes of a relativistic superfluid neutron star}

\section{Gualtieri, E. M. Kantor, M. E. Gusakov, A. I. Chugunov}

Neutron stars are among the main expected sources for gravitational wave detectors. They would provide invaluable information on the behaviour of matter at the extreme conditions occurring in their core. A very promising way to get information on the NS interior consists in looking to their proper oscillation modes, the so-called quasi-normal modes (QNMs). Once GWs from an oscillating NS will be detected, we will know the frequencies and damping times of its QNMs, which carry the imprint of the properties of NS matter.

To this aim, we need to model - in a general relativistic framework - NS oscillations, taking into account all relevant features of the matter composing the NS. However, theoretical computations and observational evidence show that neutrons and protons in NS cores are superfluid. Present computations of NS QNMs do not take into account superfluidity, or include it under strong assumptions (such as assuming zero temperature).

Using the approach introduced in [14], we studied NS oscillations taking into account both general relativity and finite temperature superfluid hydrodynamics. In [15] we have considered the limit in which superfluid degrees 
of freedom are decoupled from non-supefluid ones; we found a new class of modes, the superfluid modes, and studied the behaviour of their frequencies and damping times as the temperature of the star changes.

We have then derived the full system of equations of stellar perturbations (a generalization of Lindblom \& Detweiler's equations), consistently describing both superfluid and non-superfluid degress of freedom. We have solved numerically these equations, finding the frequencies and the damping times of superfluid and non-superfluid modes, at different values of the temperature.

\section{How to test the Kerr hypothesis?}

\section{Georgios Lukes-Gerakopoulos}

We expect that a compact object with mass greater than three solar masses is a Kerr black hole. This conjecture is known as the Kerr black hole hypothesis, and it has yet to be confirmed by observational data. In order to test this hypothesis non-Kerr spacetimes which are perturbations of the Kerr spacetime are employed. The astrophysical setup for the tests we proposed [16] is an extreme-mass-ratio inspiral (EMRI) of a stellar compact object moving into a supermassive black hole, which lies at the center of a galaxy. Our approach is based on the fact that the Kerr spacetime corresponds to an integrable system, thus any sign of non-integrability would mean that the spacetime around the central object is not described by the Kerr metric. In order to find signs of non-integrability, frequency analysis techniques can be applied on the data which will be collected by low frequency gravitational wave detectors like LISA. We have suggested two such signs. The first is the "beacon effect" of stickiness; a chaotic orbit sticks near a regular orbit and mimics the characteristic frequencies of that orbit, then moves into the chaotic sea where the signal corresponds to noise, and then returns back near to a regular orbit, and so on. Just like getting a signal from a beacon characteristic frequencies will appear and disappear. The second is the resonance effect; in this case a compact object inspiraling into a non-Kerr spacetime crosses a Birkhoff chain, which is a feature of non-integrable systems; during such crossing a pair of characteristic frequencies remain in resonance, producing a distinct plateau in the frequency ratio. Thus, if we get such signs of non-integrability, then we will have strong indications that the background is not a Kerr spacetime.

An interesting question about non-Kerr spacetimes is whether all of them are non-integrable or if there might be cases of integrable non-Kerr spacetimes. Brink, in a series of papers [17], tried to answer this question, but unfortunately she used two spacetimes for her work which were proven to be non-integrable (see e.g., [16] for the Manko-Novikov spacetime and [18] for the Zipoy-Voorhees spacetime). Another approach to the problem showed that if we impose a Carter-like type of integrability to a perturbed Kerr spacetime, then we arrive at solutions which do not satisfy the Einstein field equations [19]. Thus, the question still remains open.

G. L-G was supported by the DFG grant SFB/Transregio 7 . 


\title{
9 The Location of Resonant Orbits in the Kerr Spacetime
}

\author{
Jeandrew Brink
}

Orbital motion in the Kerr metric is characterized by three frequencies: the rotational frequency $\omega_{\phi}$ is augmented by two libration type frequencies $\omega_{r}$ and $\omega_{\theta}$. In the discussion that follows we will refer to the situation when the two libration frequencies occur in rational ratios $\omega_{r} / \omega_{\theta}=n / m$ where $n$ and $m$ are integers, as an orbital resonance. The location of resonant orbits in the Kerr metric have observable astrophysical implications for EMRIs around a supermassive black hole. If during the inspiral, the orbital frequencies of the inspiralling object become resonant an observable phase shift in the emitted gravitational radiation will take place. The location of resonant orbits also indicate which geodesic orbits are most likely to become chaotic in the event that the central object is non-Kerr or if the orbiting particle has non-zero spin. The location of resonant orbits may further serve as a plausible explanation for the observed frequencies in certain quasi-periodic oscillations measured in X-ray spectra of several black-hole candidates.

J. Brink, M. Geyer and T. Hinderer [20] presented an elegant means of completely characterizing the location and properties of resonant orbits in the Kerr spacetime for all possible values of mass, spin and orbital parameters. They presented a number of easily evaluated formula for the spatial and frequency dependence of resonant phenomenon that will simplify the task of making qualitative statements about the astrophysical system should resonant behaviour be observed. Low order $(n+m)$ value resonances that are expected to have the greatest observable impact are clustered within 50 Schwarzschild radii $R_{s}$ of the black hole horizon. It was further suggested that resonant phenomenon may result in density fluctuations on the accretion disk around the black-hole, possibly creating a Saturn ring-like structure. The typical timescales for low order resonances around the Galactic Center $\left(\operatorname{Sgr} \mathrm{A}^{*}\right)$ are $\sim 1 \mathrm{hr}$ and the resonances occur $\sim 50 \mu$ as from the black-hole. A particularly exciting prospect is that within the next 10 years, VLBI measurements using radio telescopes will attain sufficient sensitivity and temporal resolution to resolve these length and time-scales around Sgr $A^{*}$.

\section{Testing the Kerr-nature of black hole candidates}

\section{Cosimo Bambi}

Astrophysical black hole candidates are thought to be the Kerr black holes predicted by general relativity, but the actual nature of these objects has still to be verified. Robust measurements of their masses can be obtained by dynamical approaches, studying the orbital motion of gas or individual stars around them. That is enough to conclude that stellar-mass black hole candidates in X-ray binary systems are too heavy to be neutron stars for any plausible matter equation of state, while super-massive black hole candidates in galactic nuclei are too heavy, compact, and old to be cluster of 
non-luminous bodies. In other words, black hole candidates are supposed to be Kerr black holes because they cannot be explained otherwise without introducing new physics. A direct confirmation of their nature would instead be desirable.

Cosimo Bambi discussed the possibility of testing the nature of astrophysical black hole candidates with the continuum-fitting and the iron line methods. These are currently the only two relatively robust approaches to probe the geometry of the space-time around these objects. With the continuumfitting method, one studies the thermal spectrum of a geometrically thin and optically thick accretion disk. In the iron line approach, one analyzes the shape of the $\mathrm{K}-\alpha$ iron line, which is intrinsically narrow in frequency, while the one observed in the spectrum of black hole candidates is broad and distorted, as a consequence of special and general relativistic effects. These techniques have been developed to estimate the spin parameter of these objects under the assumption of Kerr background, and more recently they have been extended to test the Kerr black hole hypothesis [21]. It turns out that it is definitively difficult to confirm the Kerr nature of black hole candidates. While some exotic alternatives can be ruled out [22], in general non-Kerr black holes can look like Kerr black holes with different spin parameter and even the analysis of the continuum-fitting method and of the iron line of the same object may not break the degeneracy between spin and possible deviations from the Kerr solution [23].

\section{Imaging numerical spacetimes and testing general relativity}

\section{Odele Straub}

We propose a new test of general relativity that relates to one of the fundamental questions in astrophysics: How can we know whether a dark and compact object is indeed a black hole as predicted by Einstein's theory of general relativity? To answer this question we simulate numerically the spacetimes of various dark and compact objects, with special attention to the properties of the compact core region of our own galaxy, Sgr A*. Using a simple yet powerful model for the immediate black hole vicinity (a gaseous ion torus) we calculate images of black hole silhouettes and accretion structures - see figure 1.

Future work will encompass numerical metrics of Hořava-Lifshitz gravity and boson stars and aims at elaborating a set of criteria according to which it will be possible to discern the different models and ultimately to analyse images of the black hole silhouettes and environment observed by means of high angular resolution VLBI, such as the GRAVITY instrument (Very Large Telescope Interferometer, 2014) and the Event Horizon Telescope (fully operational ca. 2020). A catalogue of different images and orbital characteristics will facilitate the analysis and interpretation of such data and enable us to confirm or refute the presence of a general relativistic black hole in the centre of our galaxy. 

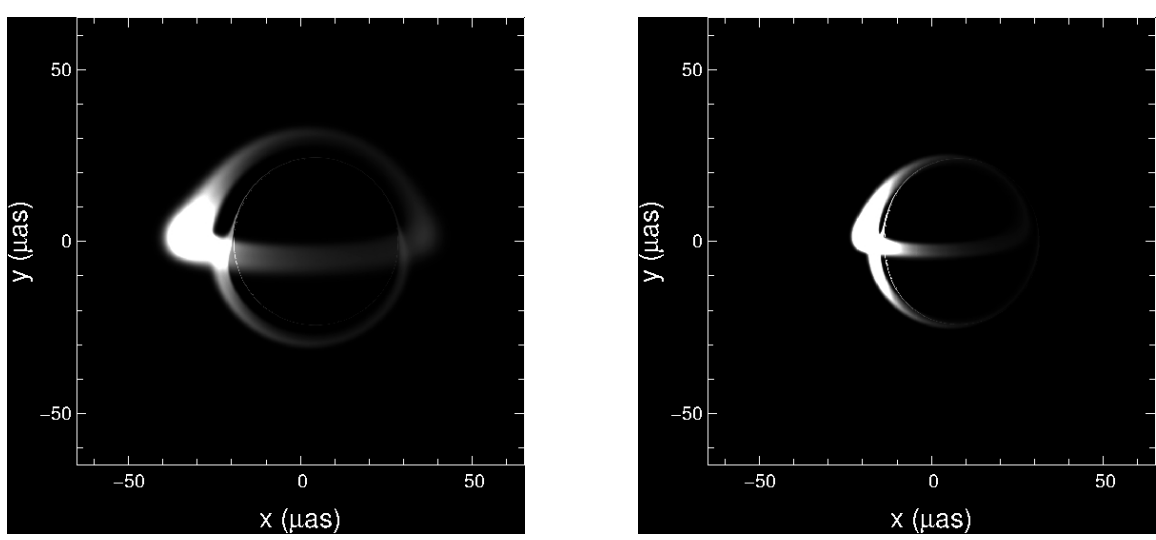

Fig. 1 The images of an optically thin ion torus around a moderately (left) and fast (right) spinning Kerr black hole as observed by an observer on Earth (see [24] for details).

\section{Observing massive black holes with extreme-mass-ratio bursts}

\section{Christopher P. L. Berry, Jonathan R. Gair}

Massive black holes (MBHs) appear to be a ubiquitous feature of galactic centres. The close correlation between the properties of MBHs and their hosts implies a coeval evolution, such that determining the history of one can inform us about the other. For MBHs, information pertaining to their formation is encoded in their mass and spin.

Gravitational wave observations of extreme-mass-ratio (EMR) systems, consisting of a stellar-mass compact object orbiting an $\mathrm{MBH}$, could provide precision measurements of $\mathrm{MBH}$ mass and spin. These would be detectable with a space-borne interferometer such as LISA or eLISA. Research into EMR signals has focussed on the later stages of the orbital evolution, the last 1-2 yr immediately preceding the plunge, by which point the orbit has nearly circularised and emits continuously within the detector's frequency band. These signals are EMR inspirals and can be observed over many orbits, allowing high signal-to-noise ratios (SNRs) to accumulate [25]. Relatively unexplored are the signals produced when the orbit is still highly eccentric, then a burst of GWs is emitted each time the compact object passes through periapsis. These EMR bursts are neither as loud as inspirals nor as informative, but could still provide a supplementary means of determining $\mathrm{MBH}$ mass and spin.

Bursts could only be detected from nearby galaxies. With LISA, bursts from systems containing MBHs of masses $M_{\bullet} \sim 10^{6}-10^{7} M_{\odot}$ and compact objects of $10 M_{\odot}$ could be detectable to distances of $R \sim 100 \mathrm{Mpc}$; for eLISA, the range is reduced by approximately an order of magnitude [27]. The Galactic Centre is the most promising source because of its proximity. A Galactic burst, assuming a $10 M_{\odot}$ compact object, could constrain the $\mathrm{MBH}$ mass to 
better than $\sim 10 \%$ and spin to better than 0.1 , if the orbit has periapse radius $r_{p} \lesssim 5 R_{s}[26]$.

An EMR system would undergo many bursts before inspiralling; the compact object could also be scattered from its orbit, meaning that it never completes the inspiral. Hence, there may be a non-negligible number of detectable bursts compared to inspirals, even though bursts are restricted to local sources. The Galactic event rate for LISA has been estimated as $\sim 0.8 \mathrm{yr}^{-1}$, meaning there is an $\sim 80 \%$ chance of observing at least one burst over a two-year mission [28]. Assuming this rate, we could expect to determine the $\mathrm{MBH}$ mass to within $\sim 1 \%$ and the spin to a precision of the order of $\sim 0.1$. This would give a useful insight into its history and, by extension, the evolution of the Galaxy.

\section{When can graviational-wave observations distinguish between black holes and neutron stars?}

Mark Hannam, Duncan Brown, Stephen Fairhurst, Chris Fryer, Ian Harry

Although gravitational-wave observations will accurately measure the chirp mass $\mathcal{M}=m_{1}^{3 / 5} m_{2}^{3 / 5}\left(m_{1}+m_{2}\right)^{-1 / 5}$ of binary mergers (where $m_{1,2}$ are component masses), a mass-ratio-spin degeneracy prevents the component masses and spins being measured accurately at likely SNRs of between 10 and $20[29,30]$. In many cases it will be difficult to determine whether the components of the binary are neutron stars or black holes [31].

However, several cases where significant results can be inferred from gravitational-wave observations were identified in [31]. For example, situations where the binary must contain (at least one) compact object that is more or less massive than anything observed to date; $\mathcal{M}<0.871 M_{\odot}$ indicates a neutron star smaller than $1 M_{\odot}, \mathcal{M}>1.741 M_{\odot}$ a neutron star larger than $2 M_{\odot}$ (if we can independently verify that it is a BNS system), and $\mathcal{M}>31.34 M_{\odot}$ a black hole larger than $35 M_{\odot}$. Observations at SNRs higher than 30 will be required to clarify the existence of the mass gap. The observation of an electromagnetic counterpart will, in certain situations, allow us to identify the system as a neutron-star-black-hole binary and, if we know the distribution of neutron-star masses, measure the black-hole mass and spin with high accuracy.

\section{The nodal precession around rotating strange stars}

Dorota Gondek-Rosinska, Wlodek Kluzniak, Nikolaos Stergioulas, Mateusz Wisniewicz

The orbital and epicyclic frequencies are of great interest in the context of X-ray observation of high-frequency variability of emission, specifically of the $\mathrm{kHz}$ quasi-periodic oscillators. Numerical calculations of rotating strange 
quark stars and their external metric show that properties of these frequencies are a result of the interplay of competing relativistic and Newtonian effects [33]. For moderately rotating massive strange stars the behavior of the epicyclic frequencies is dominated by general-relativistic effects and is similar to those of rotating neutron stars and of prograde orbits in the Kerr metric of slowly spinning black holes. However, for rapidly rotating strange stars a qualitatively new effect appears for prograde orbits - the vertical epicyclic frequency becomes larger than the orbital frequency. This is a non-relativistic effect of oblateness, known from a study of Maclaurin spheroids [32], which were verified in a calculation of the orbital and epicyclic frequencies of low mass strange quark stars [33].

\title{
15 Rossby Wave Instability as a model of microquasars high-frequency quasi-periodic oscillations (HFQPO)
}

\begin{abstract}
F. H. Vincent, P. Varniere, H. Meheut, T. Paumard, G. Torok, M. Wildner
Our work develops the Rossby Wave Instability (RWI) scenario for HFQPO, first introduced by [34]. The RWI is a hydrodynamical instability that develops in an accretion disk when the radial epicyclic frequency profile shows an extremum. Such an extremum exists in the Kerr metric at some radius $r_{0}$, close to the last stable orbit. We show that the triggering of the RWI leads to a modulation of the light curve in agreement with observed data. The HFQPO scenario may then be the following: when the inner radius of the accretion disk becomes smaller than $r_{0}$, the instability grows and leads to HFQPOs; as the inner radius evolves, it will get bigger than $r_{0}$ and the instability is quenched. This scenario leads to a natural cycle of HFQPOs that is directly linked to the evolution of the disk's inner radius. We also showed a very first simulation of a LOFT HFQPO light curve in the framework of the RWI model. Our aim is to study the ability of this future X-ray satellite to constrain our scenario.
\end{abstract}

\section{Radial accretion flows on arbitrary spherically symmetric static spacetimes}

\section{Eliana Chaverra}

We analyze the radial accretion of matter onto a static, spherically symmetric black hole background which is not necessarily Schwarzschild. Therefore, our discussion includes black hole metrics that arise in alternative theories of gravity, or as solutions of Einstein's field equations in the presence of matter fields, like dark matter, for instance. Modeling the ambient matter surrounding the black hole by a relativistic perfect fluid with an equation of state satisfying specific conditions, we reformulate the accretion problem as a dynamical system. We demonstrate that our assumptions on the metric and the fluid imply the existence of a unique critical hyperbolic point of this system. Physically, the critical point is the sonic point, where the flow's 
velocity, as measured by a static observer, is equal in magnitude to the local sound speed. Generalizing previous work by Michel [35] we prove that, for a given particle density number at in infinity, there exists a unique radial, steady-state accretion flow which is regular at the horizon.

We also compute the accretion rate for a polytropic fluid and show its dependency on the background geometry. To this end, we assume that the sound speed in the asymptotic region is much smaller than the speed of light, and the polytropic index $\gamma$ lies in the range $1<\gamma \leq 5 / 3$. The dependency of the compression rate on the background geometry is also analyzed. These results will be presented in [36].

\section{Gravitational Waves from Oscillon Preheating}

\section{Shuang-Yong Zhou}

The Universe at the end of inflation is very cold and has to be reheated to give way to the hot Big Bang. This may occur via a far-from-equilibrium process called preheating, where particles are explosively produced via a resonant phenomenon. Many interesting non-perturbative field theory phenomena may take place during preheating, including formation of quasi-solitons such as oscillons.

Oscillons are long-lived, localized excitations of nonlinear scalar fields which may be copiously produced during preheating, leading to a possible oscillon-dominated phase in the early Universe. For example, this can happen after axion monodromy inflation [37], on which we run our simulations. We investigate the stochastic gravitational-wave background associated with an oscillon-dominated phase [38]. An isolated oscillon is spherically symmetric and does not radiate GWs, and we show that the flux of gravitational radiation generated between oscillons is also small. However, a significant stochastic GW background may be generated during preheating itself (i.e., when oscillons are forming), and in this case the characteristic size of the oscillons is imprinted on the GW power spectrum, which has multiple, distinct peaks.

\section{References}

1. Bennett, M. F., van Eysden, C. A. \& Melatos, A., MNRAS 409, 1705 (2010)

2. Lasky, P. D., Bennett, M. F. \& Melatos, A., PRD 87, 063004 (2013)

3. Melatos, A., ApJ, 761, 32 (2012)

4. van Eysden, C. A. and Melatos, A., MNRAS, 409, 1253 (2010)

5. L. Sadeghian, F. Ferrer and C. M. Will, Phys. Rev. D 88, 063522 (2013) (arXiv:1305.2619).

6. P. Gondolo and J. Silk, Phys. Rev. Lett. 83, 1719 (1999) [astro-ph/9906391].

7. K. Yagi and N. Yunes, Science 341, 365 (2013)

8. K. Yagi and N. Yunes, Phys. Rev. D 88, 023009 (2013)

9. Morozova V. S., Ahmedov B. J. and Zanotti O., Mon. Not. R. Astron. Soc., 408, 490-502 (2010)

10. Morozova V. S., Ahmedov B. J. and Zanotti O., Mon. Not. R. Astron. Soc., 419, 2147-2155 (2012). 
11. Ansorg M., Gondek-Rosinska D., Villan L., MNRAS, 396, 2359 (2009)

12. Gondek-Rosinska D., Kowalska I., Villain L., Ansorg M., in preparation (2013)

13. Clemens J. C., Rosen R., Astrophys. J., 609, 340-352 (2004).

14. M.E. Gusakov, E.M. Kantor, Phys. Rev. D83, 081304(R) (2011)

15. M.E. Gusakov, E.M. Kantor, A.I. Chugunov, L. Gualtieri, Mon. Not. Roy. Astron. Soc. 428, 1518 (2013)

16. T. A. Apostolatos, G. Lukes-Gerakopoulos and G. Contopoulos, Phys. Rev. Lett. 103, 111101 (2009); G. Lukes-Gerakopoulos, T. A. Apostolatos and G. Contopoulos, Phys. Rev. D 81, 124005 (2010)

17. J. Brink, Phys. Rev. D 78, 102002 (2008); Phys. Rev. D 81022001 (2010); Phys. Rev. D 81022002 (2010); Phys. Rev. D 84, 104015 (2011)

18. G. Lukes-Gerakopoulos, Phys. Rev. D 86, 044013 (2012); A. J. Maciejewski, M. Przybylska, and T. Stachowiak, Phys. Rev. D 88, 064003 (2013)

19. S. J Vigeland, N. Yunes and L. C. Stein, Phys. Rev. D 83, 104027 (2011)

20. Jeandrew Brink, Marisa Geyer, Tanja Hinderer, arXiv:1304.0330

21. C. Bambi, Astrophys. J. 761174 (2012); Phys. Rev. D 87023007 (2012).

22. C. Bambi, Phys. Rev. D 87084039 (2013); C. Bambi and D. Malafarina, Phys. Rev. D 88064022 (2013).

23. C. Bambi, JCAP 1308055 (2013).

24. O. Straub et al, A\&A, 543, A83 (2012)

25. P. Amaro-Seoane et al., Classical and Quantum Gravity 24, R113 (2007)

26. C.P.L. Berry, J.R.Gair, Mon. Not. Roy. Astron. Soc. 429, 589 (2013)

27. C.P.L. Berry, J.R.Gair, Mon. Not. Roy. Astron. Soc. 433, 3572 (2013)

28. C.P.L. Berry, J.R.Gair, Mon. Not. Roy. Astron. Soc. 435, 3521 (2013)

29. C. Cutler and E.E. Flanagan, Phys.Rev. D 49, 2658 (1994).

30. E. Baird, S. Fairhurst, M. Hannam, E. Murphy, Phys.Rev. D 87024035 (2013).

31. M. Hannam, D. Brown, S. Fairhurst, C. Fryer, I. Harry, Ap. J. Lett. 766 L14 (2013).

32. Kluzniak, W. \& Rosinska, D., MNRAS, 434, 2825 (2013)

33. Gondek-Rosinska, D., Kluzniak, W., Stergioulas, N., Wisniewicz,M., in prep.

34. Tagger, M. \& Varniere, P., ApJ, 652, 1457 (2006)

35. F.C. Michel, Astrophysics and Space Science, 15, 153 (1972).

36. E. Chaverra and O. Sarbach, in preparation.

37. M. A. Amin, R. Easther, H. Finkel, R. Flauger and M. P. Hertzberg, Phys. Rev. Lett. 108, 241302 (2012) [arXiv:1106.3335 [astro-ph.CO]].

38. S. - Y. Zhou, E. J. Copeland, R. Easther, H. Finkel, Z. -G. Mou and P. M. Saffin, JHEP 1310, 026 (2013) [arXiv:1304.6094 [astro-ph.CO]]. 


\section{University Library}

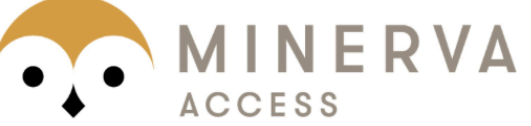

A gateway to Melbourne's research publications

Minerva Access is the Institutional Repository of The University of Melbourne

\section{Author/s:}

Mandel, I;Miller, MC;Ahmedov, BJ;Bambi, C;Berry, CPL;Brink, J;Brown, D;Chaverra, E;Chugunov, Al;Fairhurst, S;Fryer, C;Gair, JR;Gondek-Rosinska, D;Gualtieri, L;Gusakov, ME;Hannam, M;Harry, I;Kantor, EM;Kluzniak, W;Kucaba, M;Lukes-Gerakopoulos, G;Meheut, H;Melatos, A;Morozova, VS;Paumard, T;Stergioulas, N;Studzinska, A;Szkudlarek, M;Straub, O;Torok, G;Varniere, P;Vincent, FH;Wisniewicz, M;Wildner, M;Will, C;Yagi, K;Zanotti, O;Zhou, S-Y

Title:

Relativistic astrophysics at GR20

Date:

2014-05-01

\section{Citation:}

Mandel, I., Miller, M. C., Ahmedov, B. J., Bambi, C., Berry, C. P. L., Brink, J., Brown, D., Chaverra, E., Chugunov, A. I., Fairhurst, S., Fryer, C., Gair, J. R., Gondek-Rosinska, D., Gualtieri, L., Gusakov, M. E., Hannam, M., Harry, I., Kantor, E. M., Kluzniak, W. ,... Zhou, S. Y. (2014). Relativistic astrophysics at GR20. GENERAL RELATIVITY AND GRAVITATION, 46 (5), https://doi.org/10.1007/s10714-014-1688-0.

Persistent Link:

http://hdl.handle.net/11343/282759 